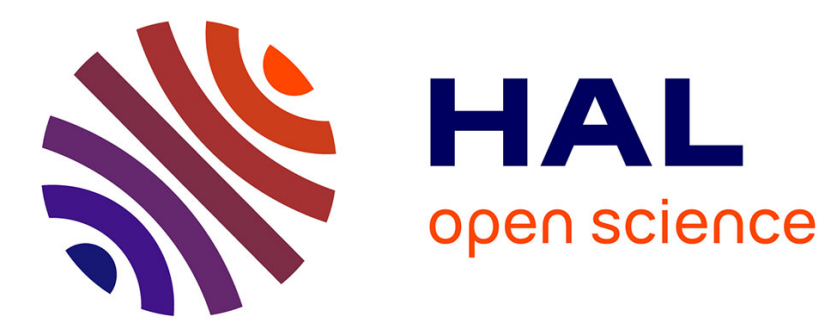

\title{
Emergence des prospectivistes 2.0, le cas des planneurs stratégiques \\ Maria Mercanti-Guérin
}

\section{To cite this version:}

Maria Mercanti-Guérin. Emergence des prospectivistes 2.0, le cas des planneurs stratégiques. Revue management \& avenir, 2008, 17 (3), pp.126. hal-02054460

\section{HAL Id: hal-02054460 \\ https://hal.science/hal-02054460}

Submitted on 1 Mar 2019

HAL is a multi-disciplinary open access archive for the deposit and dissemination of scientific research documents, whether they are published or not. The documents may come from teaching and research institutions in France or abroad, or from public or private research centers.
L'archive ouverte pluridisciplinaire HAL, est destinée au dépôt et à la diffusion de documents scientifiques de niveau recherche, publiés ou non, émanant des établissements d'enseignement et de recherche français ou étrangers, des laboratoires publics ou privés. 


\title{
Emergence des prospectivistes 2.0, le cas des planneurs stratégiques
}

\author{
Maria Mercanti-Guérin
}

Docteur en Sciences de Gestion

Centre de Recherche DMSP

DRM (CNRS UMR 7088)

Université Paris Dauphine

Maître de Conférences

Université d'Evry Val d'Essonne

Adresse : UFR SSG, 02 rue du Facteur Cheval, 91000 Evry

Téléphone : 01-46-64-06-62

Mail : maria.guerin@laposte.net 


\title{
Emergence des prospectivistes 2.0, le cas des planneurs stratégiques
}

\section{Résumé}

Emergence des prospectivistes 2.0, le cas des planneurs stratégiques

L'objectif de cette recherche est de montrer comment les sites de communautés virtuelles dédiés au planning stratégique mettent en place à travers le Web de nouveaux outils de prospective marketing. Dans une première partie, nous analyserons la fonction de planneur stratégique : origine du métier et évolutions. Dans une seconde partie, nous expliciterons les caractéristiques du Web 2.0. Un accent particulier sera mis sur ses liens avec le marketing et la communication. Dans une troisième partie, nous montrerons à travers une analyse de contenu des sites de communautés virtuelles animés par des planneurs comment l'utilisation du Web 2.0 permet de faire évoluer les outils de prospective consacrés à l'écoute des marchés.

Mots clé : planneur stratégique, Web 2.0, blogs, prospective

\begin{abstract}
The emergence of 2.0 "prospectivists", the case of account planners

The objective of this research is to show how the sites of virtual communities dedicated to account planning implement through the Web news tools to generate knowledge about marketing trends. In the first part, we will analyze the role of account planner: origin, function and evolution. In the second part, we will explain the evolving technology and characteristics of Web 2.0. Particular emphasis will be placed on the links between Web 2.0 and marketing and communication. In a third part, we will show through a content analysis of the sites of virtual communities led by planners how the use of Web 2.0 allows developing new marketing insight tools.
\end{abstract}

Key words: account planner, Web 2.0, "prospective" 


\section{Emergence des prospectivistes 2.0, le cas des planneurs stratégiques}

Le métier de planneur stratégique est un métier en pleine évolution. Il est directement touché par l'évolution des technologies de l'information qui redessine son rôle et ses fonctions au sein des agences de communication. L'objectif de cette recherche est de montrer comment les sites de communautés virtuelles dédiés au planning stratégique mettent en place à travers le Web de nouveaux outils de prospective. Dans une première partie, nous analyserons la fonction de planneur stratégique : origine du métier et évolution. Un accent particulier sera mis sur le rôle central de la prospective dans la création publicitaire et l'apparition du concept de prospective opérationnelle. Dans une deuxième partie, nous référencerons les différentes fonctions et outils applicatifs du Web 2.0. Dans une dernière partie, nous montrerons à travers une analyse de contenu des sites de communautés virtuelles animés par des planneurs comment l'utilisation du Web 2.0 permet de faire évoluer les outils de prospective et l'esprit de cette dernière.

\section{La fonction de planneur stratégique : origine du métier, évolutions et communauté}

\section{1 Origine du métier et évolutions}

La littérature consacrée au planning stratégique est assez limitée, car elle porte sur un métier relativement récent, né il y a 40 ans de la nécessité d'établir un pont entre création publicitaire, consommateurs et annonceurs. Issu de la fusion de deux professions, les média-planneurs et les responsables de compte, le planneur stratégique (en anglais Account Planner) est l'équivalent en agence du responsable du département études, marketing et prospective chez l'annonceur. "C'est un observateur doublé d'un enquêteur et d'un analyste» (Boyer et Scouarnec, 2006). Né au Royaume Uni en 1965, le planning stratégique s'impose comme métier à part entière en 1980 aux Etats-Unis. Sa relative récence explique le nombre restreint de ses membres, environ 1000 aux USA en 1999 et 200 en France en 2007 selon certaines estimations. Cette petite communauté d'experts a, de ce fait, un fort esprit communautaire renforcé par le réseau mondial mis en place progressivement, l'Account Planning Group ou APG, destiné à promouvoir le métier de planneur. Néanmoins, sa double fonction de prospectiviste et de stratège est aujourd'hui contestée. Il existe en effet une remise en cause du rôle de prospectiviste ou futurologue accordé au planneur stratégique dans les années 80 . Cette remise en cause se fait au bénéfice d'une importance plus grande donnée à sa fonction purement stratégique. Ce recentrage ne supprime pas le recours à la prospective comme outil premier de travail du planneur stratégique. Il en change l'esprit en favorisant l'émergence du concept de prospective opérationnelle.

Souvent défini comme un «tendanceur», le planneur stratégique a modifié son approche de la prospective. Il s'appuie sur cette dernière non plus pour prévoir des modes mais pour mieux comprendre et détecter des phénomènes émergents qui en quelques mois deviennent des tendances lourdes. La prospective devient un outil utilisé pour construire les plateformes de communication des marques et sélectionner les idées ou concepts sources de différenciation. Son utilité est d'orienter les discours publicitaires vers les pistes créatives les plus pertinentes pour les marques. Dans ce cadre, la prospective opérationnelle est un moyen de détection d'un contexte de mutations, définition d'une position (celle de l'entreprise face à ces mutations), et élaboration d'un projet stratégique permettant de tirer partie au mieux de ces mutations. La nécessité de lier prospective et opérationnalité est en grande partie due à une certaine mise à distance des planneurs par la communauté publicitaire : suppression de postes dans de grandes agences, mise à l'écart sur certains budgets pour lesquels créatifs et commerciaux travaillent en direct sans recours au planneur (Morisson et al., 2003), méfiance pour certaines agences françaises envers des méthodes de travail jugées typiquement anglo-saxonnes. 
Tableau 1 : l'évolution du métier de planneur stratégique (1986-2001) : réflexions de planneurs, Source: adapté de «the account planning group » (2007)

\begin{tabular}{|c|c|c|c|}
\hline & $\begin{array}{l}\text { Le métier de } \\
\text { planneur }\end{array}$ & Les compétences du planneur & L'avenir du planning stratégique \\
\hline $\begin{array}{l}\text { La vision de } \\
1986 \text { des } \\
\text { planneurs } \\
\text { sur leur } \\
\text { métier }\end{array}$ & $\begin{array}{l}\text { Représenter le } \\
\text { consommateur à } \\
\text { chaque stage de } \\
\text { développement } \\
\text { de la création } \\
\text { publicitaire et } \\
\text { imposer la } \\
\text { vision du } \\
\text { consommateur à } \\
\text { l'agence et à } \\
\text { l'annonceur }\end{array}$ & $\begin{array}{l}\text { Savoir décrypter les études de marché, tracking, usage et } \\
\text { attitude } \\
\text { Réaliser des études ad hoc pour les clients de l'agence } \\
\text { notamment dans le cadre des nouveaux concepts produit } \\
\text { Traduire les chiffres en idées créatives } \\
\text { Comprendre le fonctionnement d'une idée créative et ses } \\
\text { différentes influences possibles sur la marque } \\
\text { Savoir synthétiser et analyser le problème de communication } \\
\text { qui peut se poser à la marque } \\
\text { Guider les créatifs dans leurs réflexions } \\
\text { Vendre les créations aux clients en justifiant et rationalisant les } \\
\text { concepts choisis } \\
\text { Superviser les pré-tests et analyser les post-tests publicitaires }\end{array}$ & $\begin{array}{l}\text { Avoir une vision stratégique et visionnaire du } \\
\text { marché et permettre à la créativité des équipes } \\
\text { de se développer dans un contexte perçu } \\
\text { comme changeant sur } 3 \text { points } \\
\text { - Demande croissante de } \\
\text { professionnalisation de la publicité de } \\
\text { la part des annonceurs (éviter l'échec) } \\
\text { - Nécessité de décrypter des tendances } \\
\text { de consommation nouvelles et } \\
\text { multiples } \\
\text { - Obligation de prendre en compte la } \\
\text { force des marques, être capable de leur } \\
\text { construire une image distinctive }\end{array}$ \\
\hline $\begin{array}{l}\text { La vision de } \\
2001 \text { des } \\
\text { planneurs } \\
\text { sur leur } \\
\text { métier }\end{array}$ & $\begin{array}{l}\text { Guider et } \\
\text { faciliter le } \\
\text { processus de } \\
\text { création en } \\
\text { s'appuyant sur } \\
\text { son expertise } \\
\text { des marchés et } \\
\text { du } \\
\text { consommateur }\end{array}$ & $\begin{array}{l}\text { Chercheur en marketing et analyste de bases de données } \\
\text { Animateur de focus groupes qualitatifs } \\
\text { Centralisateur de l'information marketing au sein de l'agence } \\
\text { Partenaire du commercial agence } \\
\text { Consultant en développement de nouveaux produits } \\
\text { « Facilitateur » créatif } \\
\text { Représentant du consommateur } \\
\text { Futurologue } \\
\text { Expert Média } \\
\text { Stratège } \\
\text { Penseur et polémiste } \\
\text { Chercheur de tendances } \\
\text { Traducteur de théories }\end{array}$ & $\begin{array}{l}\text { Condamner à disparaître : } \\
\text { Car l'expertise marketing est du côté des } \\
\text { annonceurs plus des agences } \\
\text { A moins de savoir proposer des solutions de } \\
\text { communication } \\
\quad \text { - Pertinentes } \\
\quad \text { - Distinctives } \\
\quad-\text { Efficaces }\end{array}$ \\
\hline
\end{tabular}




\subsection{Nature de la communauté des planneurs : une communauté de pratique encore en devenir}

Parmi les typologies reconnues des communautés de savoir virtuel, la communauté regroupant les planneurs stratégiques francophones peut être considérée comme une communauté métier. L'objectif principal d'une communauté métier est d'approfondir le degré de savoir-faire et d'expertise de ses membres sur une thématique donnée grâce à l'interaction (Wenger et al., 2002).

La nature de cette communauté métier a une typologie particulière dans le sens où elle est à la fois inter-organisationnelle et ayant pour ambition de représenter une plateforme de regroupement de nombreux métiers : planneurs, créatifs, annonceurs, psychologues, médiaplanneurs...Ainsi, elle rassemble à elle seule les trois types de communauté de pratique distingués par Probst et Borzillo (2007) :

- Une communauté de pratique stratégique fondée sur la création de synergies entre ses membres afin de développer un savoir innovant

- Une communauté de pratique fondée sur l'excellence opérationnelle dont l'objectif est de sélectionner les pratiques les plus efficaces dans l'approche de son métier

- Une communauté de pratique fondée sur l'espace social et productif offrant à ses membres un lieu convivial et sécurisant

Néanmoins, cette communauté de pratique apparaît comme une communauté aux contours flous encore en devenir dont l'étude se heurte à un certain nombre de difficultés. La première difficulté réside dans la reconstitution de la communauté des planneurs français sur le Web. En effet, les leaders reconnus de cette communauté sont pratiquement tous anglo-saxons. Leur Wiki Plannersphere témoigne de leur volonté d'organisation en un réseau social structuré permettant l'échange d'information et l'identification de ses membres. La deuxième difficulté réside dans le peu de liens que la communauté des planneurs stratégiques français entretient en son sein. La communauté française souffre d'une scission entre planneurs, ayant un profil plutôt sociologique, et dont l'ouverture sur d'autres communautés est essentiellement axée sur celle des créatifs et planneurs, ayant un profil plutôt marketing, et dont les centres d'intérêt rejoignent ceux des consultants en nouvelles technologies, managers projets, spécialistes de la veille technologique ${ }^{1}$. Néanmoins, le repérage des principaux acteurs de cette communauté et de leurs interactions a été facilité par le fait qu'il existe au sein de la communauté des planneurs stratégiques français une prise de conscience progressive de leur manque de structure et de visibilité. Un Wiki français est d'ailleurs à l'étude. L'émergence du Web 2.0 tout en soulignant les fractures de cette communauté représente une réelle opportunité pour cette dernière de progresser en expertise, notamment dans le domaine de la prospective opérationnelle.

\section{L'utilisation du Web 2.0 comme outil de prospective appliqué au marketing et à la communication}

Né au cours de l'été 2004 d'un brainstorming rassemblant un journaliste et deux acteurs de l'industrie des médias, le Web 2.0 peut se définir comme un réseau social dont la souplesse et les possibilités accrues de création, diffusion et communication permettent à l'utilisateur de

\footnotetext{
${ }^{1}$ Nous avons reconstitué à travers le blogroll d'un site son arbre de diffusion. Il apparaît que près de $50 \%$ de sa communauté amie est constituée de créatifs, plus spécifiquement de directeurs artistiques. A peine $10 \%$ est constituée de planneurs. Le reste se partage entre des concepteurs rédacteurs et des free-lances qui font essentiellement du conseil en nouvelles technologies. Concernant les arbres de diffusion, nous recommandons le blog de philippe Gambette (http : //philippe.gambette.free.fr/Blog/FLists) qui grâce à un algorithme spécifique a réussi à reconstituer la blogosphère française (F-list). La F-list intègre les meilleurs blogs francophones (Internet, Web 2.0, technos, design...). Le principe est de faire circuler de blog en blog la liste afin de contribuer à son enrichissement.
} 
devenir «co-créateur de nouvelles applications en ligne, initiant souvent de façon collective ou communautaire de nouvelles formes de réactions numériques » (Asselin pour Digimind, 2007). Les sept principes du Web 2.0 peuvent se résumer ainsi :

Tableau 2 : Les sept principes du Web 2.0

Source : adapté de O'Reilly (2005), repris par Asselin pour Digimind (2007)

\begin{tabular}{|c|}
\hline Une plate-forme de services \\
\hline Des internautes co-développeurs des applications \\
\hline Une amélioration du service qui croît avec le nombre d'utilisateurs \\
\hline Des données riches et des données libres « sans propriétaire » \\
\hline L'importance de l'intelligence collective dans la production \\
\hline La mise en place d'interfaces souples et légères \\
\hline L'affranchissement du PC et son utilisation sur des objets nomades (portable, lecteur audio) \\
\hline
\end{tabular}

Ainsi, les caractéristiques du Web 2.0 renforcent le travail collaboratif en assouplissant et simplifiant la production et diffusion de l'information. Ces nouvelles formes de relation sont rendues possibles par un certain nombre d'outils largement utilisés par les communautés virtuelles :

- Les outils de partage et de collaboration : bookmarks sociaux, réseaux sociaux, multimédia, syndication

- Les outils de diffusion : wikis, blogs et flux RSS

- Les outils de recherche et de collecte de l'information : blogs et flux RSS, tags, moteurs de recherche collaboratifs, bases de données, outils sémantiques

- Les outils d'étude: bulletin board et focus group online, web-reporting, géomarketing à travers les applications composites, analyse des blogs introspectifs

- Les outils de surveillance et de veille: détection et agrégation de flux RSS, utilisation d'outils type Digimind

La nouveauté de ces outils est contestée par bon nombre d'experts pour qui le Web 2.0 n'est qu'un enrichissement des interfaces utilisateurs fondées sur les technologies éprouvées du Web 1.0. Néanmoins, sa fonction de partage des connaissances, d'identification des sources et d'évaluation des productions par les internautes eux-mêmes en fait un espace où le marketing et la communication sont omniprésents, ses fonctionnalités se mariant aux nouveaux concepts de ciblage et de segmentation : logique de CRM (customer relationship management), buzz marketing ou marketing du bouche à oreille, marketing de la valeur client, dialogue avec la marque, blogs corporate, communication interactive. De ce fait, la prospective marketing devient un champ privilégié de recherche sur le Web 2.0. 
Tableau 3 : les principaux outils du Web 2.0

\begin{tabular}{|c|c|c|}
\hline \multirow[t]{2}{*}{$\begin{array}{l}\text { Outils de } \\
\text { partage et de } \\
\text { collaboration }\end{array}$} & $\begin{array}{l}\text { Bookmarks sociaux: mise en relation d'individus } \\
\text { partageant les mêmes centres d'intérêt personnels ou } \\
\text { professionnels }\end{array}$ & $\begin{array}{l}\text { Linkedin } \\
\text { Viadeo }\end{array}$ \\
\hline & Syndication $(c . f$. flux RSS) & $R S S 2.0$ \\
\hline \multirow[t]{3}{*}{$\begin{array}{l}\text { Outils de } \\
\text { diffusion }\end{array}$} & $\begin{array}{l}\text { Wikis : site de mutualisation des connaissances dont } \\
\text { les contributeurs sont généralement anonymes }\end{array}$ & Wikipédia \\
\hline & $\begin{array}{l}\text { Blogs: journal personnel disponible sur le Web } \\
\text { comprenant } \\
\text { - Un blogroll, liste de liens vers d'autres blogs } \\
\text { choisis par l'auteur } \\
\text { - Des billets, messages et articles du plus ou } \\
\text { moins récent identifiables via une adresse } \\
\text { (URL) précise } \\
\text { - Des catégories, classement des billets par } \\
\text { thématique et des archives } \\
\text { - Des commentaires des visiteurs sur les billets } \\
\text { - Des trackbacks permettant de connaître les } \\
\text { sources ayant cité le billet du blog } \\
\text { - Des Podcasts, fichiers audio au format } \\
\text { numérique }\end{array}$ & \\
\hline & $\begin{array}{l}\text { Flux RSS ou really simple syndication, permet } \\
\text { d'extraire automatiquement d'un blog un contenu } \\
\text { régulièrement mis à jour }\end{array}$ & $\begin{array}{l}\text { RSS } \\
\text { Atom }\end{array}$ \\
\hline $\begin{array}{l}\text { Outils de } \\
\text { recherche }\end{array}$ & $\begin{array}{l}\text { Tags, mot clé défini par le blogueur de façon à décrire } \\
\text { un document (texte, vidéo, photo...), indexation dite } \\
\text { subjective } \\
\text { Souvent visualisé en nuages de tags sur les blogs et } \\
\text { utilisé à la place des mots clé dans les moteurs de } \\
\text { répertoires de blogs }\end{array}$ & $\begin{array}{l}\text { Googleblog } \\
\text { search } \\
\text { Digg } \\
\text { Wikio }\end{array}$ \\
\hline $\begin{array}{l}\text { Outils } \\
\text { d'études }\end{array}$ & $\begin{array}{l}\text { Applications composites ou Mashup mixant plusieurs } \\
\text { sources et permettant d'offrir de nouveaux services }\end{array}$ & $\begin{array}{l}\text { Google } \\
\text { Earth }\end{array}$ \\
\hline $\begin{array}{l}\text { Outils de } \\
\text { surveillance } \\
\text { et de veille } \\
\end{array}$ & $\begin{array}{l}\text { Flux RSS } \\
\text { Agents aspirateurs (recherche automatisée) }\end{array}$ & $\begin{array}{l}\text { Digimind } \\
\text { Opinion } \\
\text { Way }\end{array}$ \\
\hline
\end{tabular}

\section{Prospective sur le Web 2.0 : le cas des planneurs stratégiques}

\subsection{Champ de recherche, méthodologie et objectifs de la recherche}

\section{3-1-1 Champ de recherche et méthodologie}

Afin de mesurer l'utilisation de ces nouveaux outils au sein d'une communauté métier, nous avons conduit notre recherche auprès de quatorze blogs animés par des planneurs stratégiques français. Il est à noter que nous avons souhaité adapter nos outils d'analyse à la configuration spécifique des blogs 2.0 . 


\begin{tabular}{|c|c|}
\hline \multicolumn{2}{|c|}{ Tableau 4 : Méthodologie de recherche } \\
\hline Population investiguée & $\begin{array}{l}\text { 14 blogs tenus par des planneurs stratégiques } \\
\text { provenant } \\
\text { - d'une recherche sur le mot-clé } \\
\text { «planneur stratégique » } \\
\text { - d'une recherche au format RSS } \\
\text { - d'une recherche sur des moteurs de } \\
\text { recherche de blogs type Google } \\
\text { Blog Search ou Blogorama } \\
\text { - d'une recherche effectuée sur les } \\
\text { Blogrolls des blogs déjà connus } \\
\text { - d'une consultation de leur Wiki } \\
\text { international, Plannersphere wiki }\end{array}$ \\
\hline Objet de l'investigation & $\begin{array}{l}\text { Déterminer quels sont les nouveaux outils et } \\
\text { pratiques } 2.0 \text { destinés à la prospective et } \\
\text { utilisés par les planneurs stratégiques } \\
\text { français }\end{array}$ \\
\hline Méthode d'investigation & $\begin{array}{l}\text { Analyse sémantique du contenu des blogs } \\
\text { sélectionnés à travers } \\
\text { - Les billets } \\
\text { - } \text { Les trackbacks } \\
-\quad \text { Les commentaires } \\
-\quad \text { Les catégories } \\
-\quad \text { Les podcasts } \\
\text { - } \quad \text { Les tags }\end{array}$ \\
\hline $\begin{array}{l}\text { Terrain d'investigation } \\
\text { http://lexeul.blogspot.com/ } \\
\text { http://bertrandespitalier.blogspot.com/ } \\
\text { http://cyrille-chaudoit.blogspot.com/ } \\
\text { http://nekid.fr/ } \\
\text { http://agencements.blogs.com/ } \\
\text { http://www.trilogicom.com/ } \\
\text { http://accountplan.ning.com/ } \\
\text { http://www.julienchavane.com/ } \\
\text { http://www.groupereflect.net/blog/ } \\
\text { http://www.cedricamiot.com/ } \\
\text { http://www.thomevincent.com/blog/ } \\
\text { http: //www.levidepoches.fr/ } \\
\text { http : //www.darkplanner.com/ } \\
\text { http: //www.lafabriquedufutur/ }\end{array}$ & \\
\hline
\end{tabular}

\section{3-1-2 Objectifs de la recherche}

Les objectifs de cette recherche sont les suivants :

- Vérifier à travers une communauté qui se destine à la prospective si cette dernière utilise les nouveaux outils mis à sa disposition sur le Web 2.0

- Etudier si les nouvelles pratiques observées modifient la communauté en son sein

- Evaluer les changements que ces outils peuvent apporter aux méthodes liées à la prospective 
Ainsi nos résultats de recherche comportent une partie organisationnelle à travers l'étude d'une plate-forme collaborative et une partie plus descriptive destinée à mesurer l'évolution des pratiques d'un prospectiviste en marketing en fonction des outils mis à sa disposition.

\subsection{Organisation des équipes de prospectivistes, l'art de faire de la prospective extra- communautaire : l'exemple du Vide-poches}

Nous avons pris l'exemple du Vide-poches comme illustration d'une plate-forme collaborative ouverte sur l'ensemble des métiers nécessaires à l'activité d'un planneur stratégique. Le Vide-poches animé par trois planneurs stratégiques a pour ambition de pratiquer la prospective à travers la mise en collaboration de différentes communautés. La prospective 2.0 est alors assimilée à «une hybridation du techno-push et du market-pull » (Seuillet in JDN Management, 2005).

Les objectifs de la plateforme sont de :

- observer, étudier, veiller et analyser les clients, les marchés et les tendances

- générer des concepts d'offre

- illustrer, scénariser, maquetter, prototyper et simuler

- tester, confronter au réel, prioriser et sélectionner les concepts en vue de leur application

Les communautés rassemblées autour du Vide-poches appartiennent au milieu des agences, des maisons de production, des annonceurs, des acteurs de la vie associative.

Le planneur stratégique se définit comme un connecteur pratiquant la pluridisciplinarité, la transversalité et l'utilisation de l'intelligence collective rendus possible par le développement du Web 2.0 défini comme «participatif et conversationnel » (La fabrique du futur, 2007). La think tank classique est remplacée par une think network. Néanmoins, cette activité de connecteur sur le Web rend possible la rencontre des différentes communautés dans la vie réelle autour de thématiques prédéfinies et jugées dans l'air du temps.

Figure 1 : Illustration d'une plate-forme collaborative, l'exemple du Vide-poches Le Vide-poches, 2007

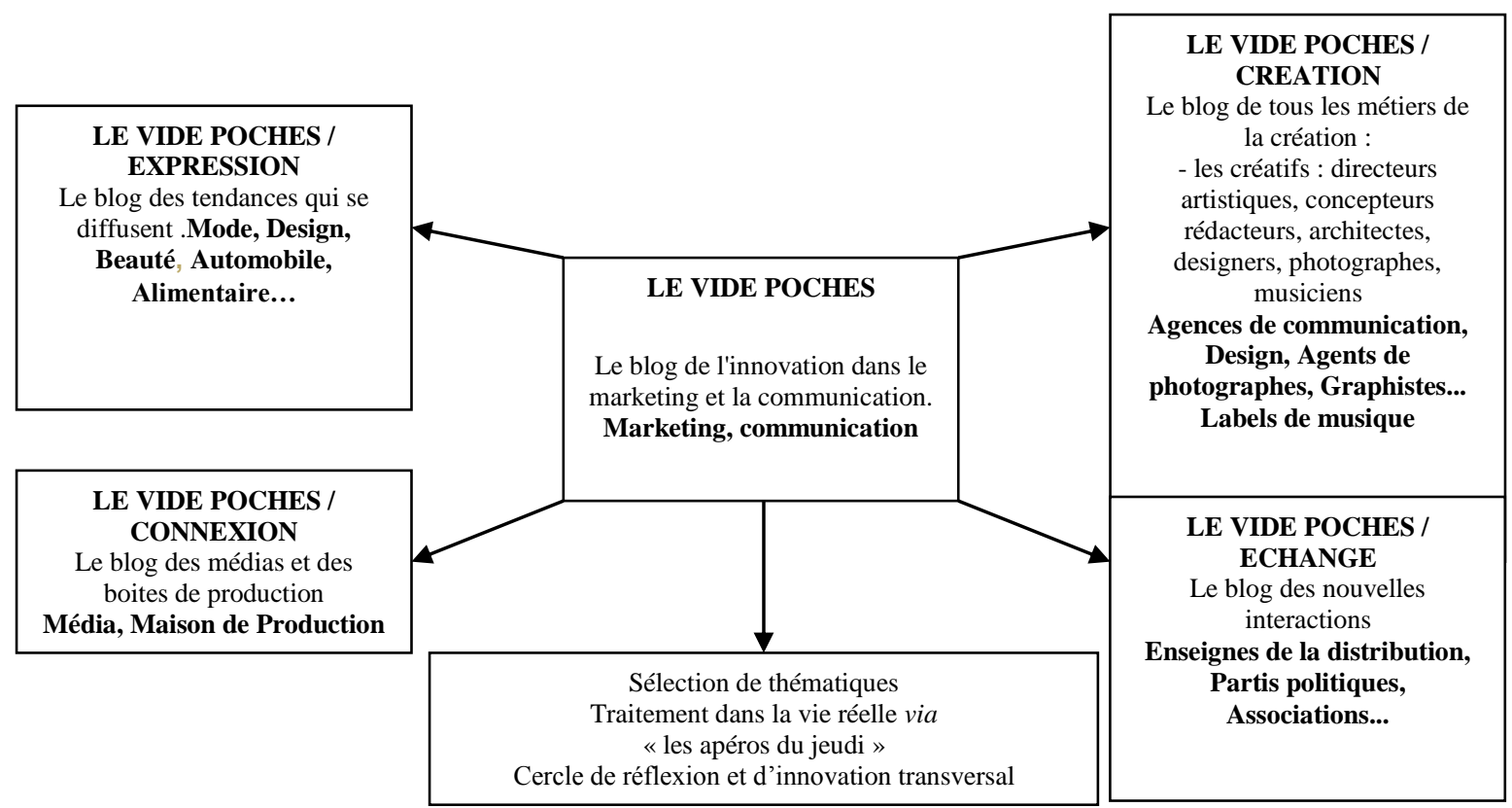


Si l'activité quotidienne du planneur stratégique a toujours été de s'ouvrir à d'autres communautés, il apparaît que le Web 2.0 facilite ce travail de connexion.

Tableau 5 : le quotidien du planneur stratégique

\begin{tabular}{|c|c|}
\hline Le planneur stratégique avant le Web 2.0 & Le planneur stratégique 2.0 \\
\hline Appartient aux réseaux des écoles ou des & Utilise son blog pour se faire connaître en \\
\hline $\begin{array}{l}\text { Appartient aux associations professionnelles } \\
\text { (marketing, média...) }\end{array}$ & $\begin{array}{l}\text { Utilise les blogs d'autres communautés pour } \\
\text { construire son expertise }\end{array}$ \\
\hline $\begin{array}{l}\text { Organise ou intervient dans des cycles de } \\
\text { séminaires ou de conférences }\end{array}$ & $\begin{array}{l}\text { Développe sa communauté } \text { (blogroll) } \\
\text { française et étrangère }\end{array}$ \\
\hline $\begin{array}{l}\text { A vécu à l'international et y a gardé des } \\
\text { contacts }\end{array}$ & $\begin{array}{l}\text { Développe sa propre agence de recherche de } \\
\text { tendance et de production d'idées }\end{array}$ \\
\hline Change régulièrement d'agence & Ecrit des billets \\
\hline Ecrit dans les journaux professionnels & Organise des podcasts \\
\hline $\begin{array}{l}\text { Se rend à des salons et à des tables rondes de } \\
\text { professionnels }\end{array}$ & \\
\hline $\begin{array}{l}\text { Doit être capable d'avoir une expertise sur } \\
\text { tous les secteurs }\end{array}$ & \\
\hline
\end{tabular}

Par ailleurs, les valeurs du Web 2.0 appliquées à la prospective marketing rejoindraient les valeurs affichées des planneurs stratégiques : partage et transparence, liberté d'expression, autonomie.

Néanmoins, la plate-forme collaborative du Vide-poches contribue également fortement à la notoriété de ses membres. Ainsi, elle apparaît comme un des premiers sites référencé par Google et Yahoo lorsque l'on tape les mots clés: planning stratégique ou planneur stratégique. Son fondateur réunit plus d'occurrences sur son nom que le président de l'APG France. Se définissant lui-même comme un leader créatif, la richesse de sa plate-forme en fait une des personnalités fortes de sa communauté. Parallèlement d'autres blogs de planneurs restent dans un anonymat revendiqué ou dans une certaine discrétion.

\subsection{Changements des pratiques de prospective : nouveaux outils et nouvel esprit}

Dans le même temps, l'utilisation de nouveaux outils change la façon de faire de la prospective et l'esprit dans laquelle elle est réalisée. Elle touche à deux domaines prioritaires pour les planneurs stratégiques : la détection des tendances et leur influence sur la perception de la marque. Elle modifie en profondeur les outils de prospective utilisés au quotidien par les planneurs stratégiques: études, cahiers de tendance, monographies de cible...

La figure suivante est un panorama des différents outils 2.0 utilisés dans un objectif de prospective par les planneurs stratégiques. Certains outils ont parfois une double fonction (sociaux ou de ressources documentaires). Ils ont été référencés selon le principe suivant :

- Analyse de l'ergonomie des blogs des planneurs stratégiques, de leur configuration et de leurs différents services de syndication

- Analyse sémantique des billets, archives et catégories mentionnant les outils de prospective utilisés, leurs fonctionnalités et leurs limites 
Figure 2 : outils prospectifs Web 2.0 et planning stratégique

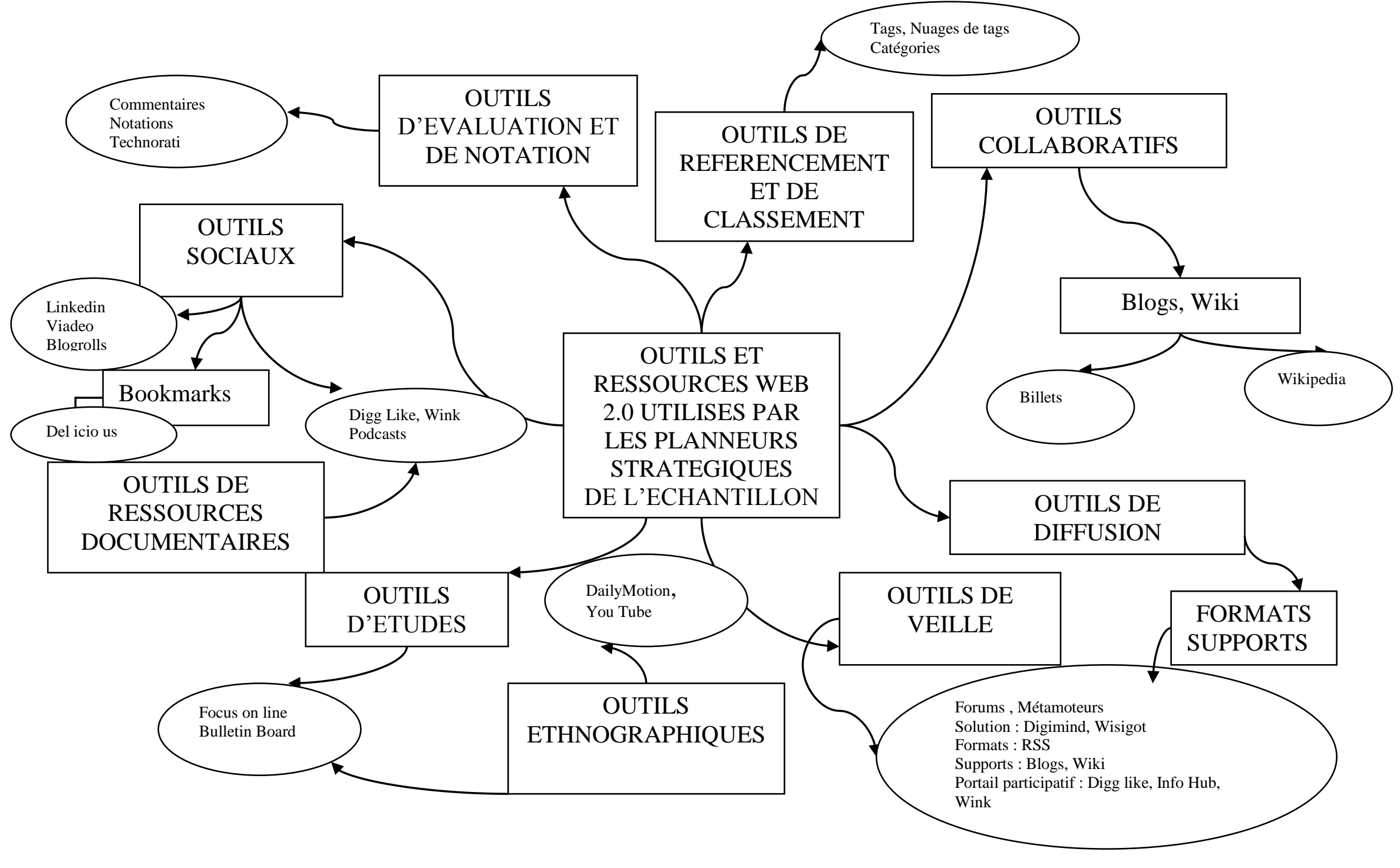




\subsubsection{Détection des tendances et gestion de la marque}

L'un des changements majeurs introduit par le Web 2.0 réside dans une nouvelle approche des populations dites avant-gardistes. La consommation de ces populations a toujours constitué un indicateur important de détection des évolutions. Grâce au Web 2.0, l'étude des blogs notamment introspectifs ouvre la possibilité de saisir l'imaginaire du consommateur innovateur et les usages émergents dont il est porteur. Désignés sous le terme de créatifs culturels (Le Vide-poches, 2007) ou d'influenceurs, ils représentent une source d'information sur les aspirations du consommateur de demain. Cette étude des cibles émergentes est pratiquée par les planneurs stratégiques en :

- analysant les requêtes tapées sur les principaux moteurs de recherche et les tags (mots des blogs)

- étudiant la lexicalisation des mots-clés dans une optique de référencement mais aussi de choix de termes de consommation

- rassemblant les contributions sur les blogs, forums, wiki, réseaux sociaux afin de suivre la réputation de la marque sur le net

- étant présent et surveillant les nouveaux mondes virtuels type Second Life afin d'y tester des nouveaux concepts de produit et de service

- construisant des panels d'avatars et non plus de consommateurs

Les objectifs futurs que les planneurs stratégiques assignent à la marque et les ambitions qu'ils nourrissent concernant l'évolution de leur métier sont les suivants (La fabrique du futur, 2007) :

- déterminer qui sont les influenceurs et quels sont leurs centres d'intérêt

- redessiner leur réseau et leur navigation à travers l'étude des bookmarks sociaux (partage des sites ou pages favoris)

- faire du consommateur, notamment le consommateur innovateur, l'ambassadeur de la marque et son propre média (Buzz Marketing, bouche à oreille)

- détecter à travers le Web les relations rationnelles et affectives que le consommateur entretient avec la marque, prémisses des comportements d'achat futurs

- trouver des idées interactives et participatives en s'appuyant sur les consommateurs innovateurs $^{2}$

- remarquer les points d'accord des internautes entre eux ou les sujets déclenchant des réactions de rejet grâce à la technique des votes et évaluations des articles, des blogs et des produits

Le Web 2.0 rend de ce fait obsolète un certain nombre d'outils tels que les cahiers de tendance. Définis comme une photographie annuelle des mouvements de la société et des phénomènes macro-sociétaux, ils sont aujourd'hui considérés comme :

- Manquant d'opérationnalité

- Insuffisant pour réaliser une prospective efficace car tenant plus de l'analyse de contexte que de la prospective

\section{3-3-2 Réalisation des études on-line et ethnographie}

Le suivi et la commande d'études en agence ont toujours fait partie des attributions des planneurs stratégiques. Se définissant comme le représentant du consommateur auprès des créatifs et des annonceurs, le planneur stratégique supervise des études essentiellement

\footnotetext{
${ }^{2}$ La campagne Levi's diffusée pour le Superbowl américain a été imaginée par un simple consommateur internaute suite à un jeu concours lancé sur le Web.
} 
qualitatives destinées à orienter le processus créatif. Deux types d'études sont rendus possibles grâce au Web 2.0 :

- Les focus groups en ligne : ces réunions de groupe sont animés par Internet et reposent sur l'utilisation de logiciels de chat spécialisés (salons virtuels, salles de réunion virtuelles)

- Le Bulletin Board ou bulletin d'information permet d'étudier sur une durée de 2 jours à 3 semaines les ressentis ou perceptions des consommateurs de façon détaillée et poussée. Les consommateurs peuvent consommer sur le long terme le produit à tester, ce qui aboutit à une visualisation du contexte naturel de consommation. La démarche ethnographique peut être complétée par les participants à qui l'on demande de prendre des photos numériques de leur univers puis de les mettre en ligne.

L'exploration ethnographique permet de détecter les concepts les plus porteurs, d'identifier et d'analyser les freins à la consommation et de tester en ligne des nouvelles idées, qui peuvent être remplacées la semaine suivante par une autre génération d'idées. Elle facilite le travail prospectif en modifiant les procédures de tests et en les rapprochant de la technique des scénarii grâce à l'injection en ligne de différents types de stimuli (vidéos, sons ...) et donne une valeur ethnographique aux tests de concept et de produit (bétatesteurs). Les études 2.0 modifient également la constitution des échantillons en ne retenant que les consommateurs les plus participatifs, impliqués et innovants. Ces techniques remettent en cause le sacro-saint principe de la représentativité de l'échantillon et privilégient le potentiel créatif des sondés.

\section{3-3-3 Un autre utilisation de l'image et de la vidéo}

Si l'ensemble des blogs tenus par des planneurs stratégiques fait une utilisation extrêmement courante de la vidéo notamment par la mise en ligne d'entretiens filmés avec des professionnels ou experts de domaines (podcasts), la recherche d'images puis de vidéos comme outils de détection de nouvelles tendances prend une autre portée sur le Web 2.0. Elle rend possible une veille axée sur le consommateur, sur un secteur ou sur les concurrents. L'image et la vidéo 2.0 peuvent constituer un outil de veille permettant de prendre en compte le contexte de la prise de vue, les tags que les internautes associent à leurs photos, les notes que recueillent photos et films (YouTube, Daily Motion). Des sites comme The Eyes have it fournissent une vue globale de la communication visuelle des secteurs de la pharmacie et de la biotechnologie.

\section{3-3-4 Une veille marketing automatisée}

La veille marketing largement pratiquée par les planneurs suit un processus extrêmement codifié en quatre étapes (Inforizon, 2007) :

- mise en place d'équations de recherche correspondant aux thématiques de veille notamment grâce à l'utilisation de métamoteurs

- évaluation de la source

- intégration au bookmark

- sélection des pages les plus susceptibles d'actualisation et réglage des paramétrages de surveillance via le RSS

- archivage à travers un système d'information veille permettant également les commentaires associés

- diffusion

Cette veille automatisée est rendue possible grâce à l'utilisation d'agents d'alerte ou d'agents aspirateur (Wysigot) 


\section{3-3-5 Web 2.0 et sites d'actualité collaborative (Digg Like) : de nouvelles ressources documentaires}

Le planneur stratégique enrichit sa réflexion prospective par l'utilisation de portails participatifs sur lesquels sont déposées des actualités proposées par les internautes. Ces catégories peuvent être divisées en thématique et bénéficient d'un modérateur. Ainsi le Digg Like consacré au Marketing, Marketingrama bénéficie d'un classement des articles les plus lus et les mieux notés (782 items à ce jour), des commentaires afférents et d'un étiquetage via un système de tags. L'étude des commentaires et des articles permet de mesurer en temps réel la popularité d'un produit mais également les souhaits non satisfaits en matière de consommation. A noter également l'émergence des cahiers blancs circulant librement sur les blogs.

\section{3-3-5 Prospective 2.0 et chaîne de création}

Une des dernières thématiques abordées par les blogs des planneurs stratégiques concerne le processus de création et son séquençage. Si la copy strategy est dans certaines agences rédigée sous forme de Wiki, les planneurs du Web 2.0 réclament de «briser la chaîne séquentielle du positionnement, brief, création pour remettre l'idée au cœur de la création » (Dumont in slideshare.net, 2007). La souplesse du Web 2.0 permet, comme nous l'avons vu précédemment, de tester presque en direct des scenarii et des concepts. Fondé sur le principe de la folksonomie $e^{3}$ ou peuplonomie, le Web 2.0 repose sur la subjectivité des internautes en terme de classification et de ce fait représente un indicateur des aspirations profondes des consommateurs et de leurs centres d'intérêt.

\section{Conclusions}

Cette recherche est une première tentative d'explication et de description des effets du Web 2.0 sur une communauté de pratique, son organisation et ses outils. Elle montre que la mutation assez radicale de ces derniers change à la fois l'esprit de la prospective en remettant (du moins dans les discours) le client au centre de la démarche et structure la communauté en lui permettant grâce à une intensification des échanges entre ses membres de prendre conscience de l'intérêt du travail collaboratif.

Elle apporte également un autre sens à des concepts tels que la prospective vue «comme une hybridation et interaction entre les imaginaires d'un vaste réseau d'individus » (Seuillet in JDN Management, 2005) nécessitant de «croiser les expertises plutôt que de les laisser s'autosatisfaire », cette expertise pouvant être une expertise consommateur.

Elle donne une autre fonction au planneur : créateur (de blogs), co-créateurs (à travers des outils collaboratifs comme Digg like) et éditorialiste, ce qui l'autorise à son tour à avoir une position subjective et personnelle plus que scientifique, mais également plus propice à l'analyse des signaux faibles.

Le Web 2.0 permet donc de :

- relier des disciplines qui cohabitent rarement (sociologie, informatique, design...) ce qui facilite le rôle de chef d'orchestre du planneur stratégique

- croiser des méthodologies complémentaires (sémiologie, observation ethnologique ou in situ...) et les confronter directement aux consommateurs innovateurs

- anticiper les tendances par une utilisation extensive des méthodes de veille

Néanmoins, il convient de mentionner un certain nombre de limites à ces nouvelles pratiques de prospective.

\footnotetext{
${ }^{3}$ Classification collaborative et subjective fondée sur les Tags
} 
- L'extrême richesse et variété d'informations rendent difficile leur assimilation opérationnelle et leur enrichissement, ce qui semble d'ailleurs être la dernière chasse gardée du planneur stratégique

- Elles introduisent un autre rapport au temps où les délais de réaction aux tendances émergentes de la société semblent se raccourcir

- Le Web 2.0 apparaît comme un catalogue de données peu structurées et son remplacement par un Web 3.0 dit Web sémantique offrant un répertoire global d'informations exploitables notamment par les machines est d'ores et déjà programmé. La réponse à des requêtes se fera plus par des solutions construites que par des listes de documents ce qui induit une prospective marketing possiblement entièrement automatisée.

Nous citerons en dernière limite les effets de groupe induits par le Web 2.0. Le premier effet de groupe réside dans le lien existant entre autorité et information (Broudoux, 2007). Parmi les différents types d'autorité, l'autorité du support donne de l'importance à l'information, ce qui induit que les blogs les plus populaires voient leurs informations plus volontiers reprises que ceux qui le sont moins. Le Web 2.0 plus qu'un outil prospectif devient un espace de prise de pouvoir pour certains membres de la communauté.

Le second effet de groupe tient aux critères d'évaluation et de notations des informations par les internautes. Ainsi, la notion même d'expertise disparaît avec une nouvelle approche plus $\mathrm{C}$ to $\mathrm{B}$ (du consommateur à l'entreprise) que $\mathrm{B}$ to $\mathrm{C}$ (de l'entreprise au consommateur). Quelle sera alors la place du planneur stratégique dans cette nouvelle configuration? 


\section{WEBOGRAPHIE}

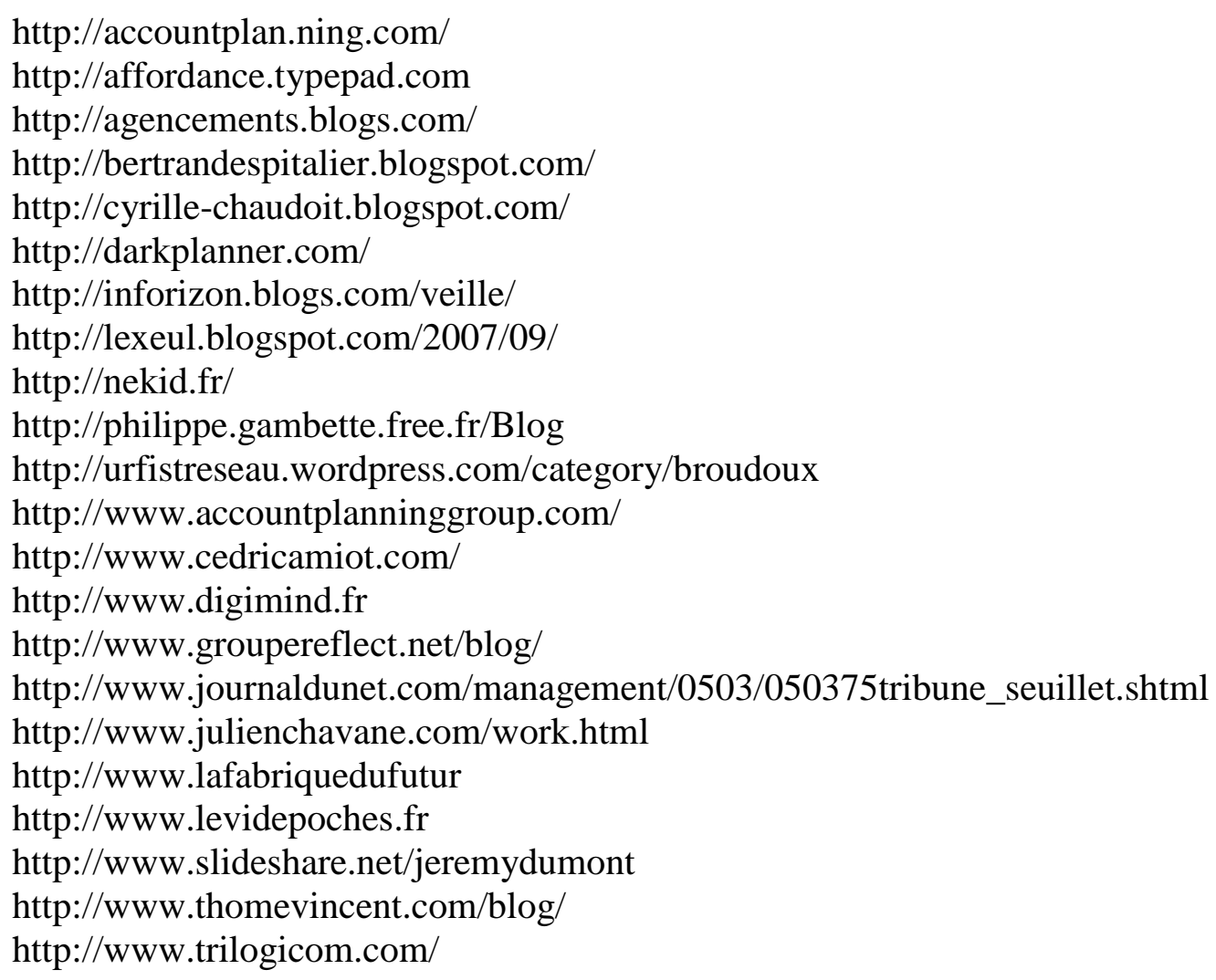

\section{BIBLIOGRAPHIE}

L. Boyer et A. Scouarnec (2006), démarche prospective métier pub/com, Observatoire des métiers de la communication

A. Margaret Morrison et E. Haley (2003), «Account Planners'view on how their work is and should be evaluated », Journal of Advertising, vol 32, p 4-16, Summer

G. Probst et S. Borzillo (2007), «Piloter les communautés de pratique avec succès », Revue française de gestion, $\mathrm{N}^{\circ}$. 170, p. 137-153, janvier

E. Wenger, R. McDermott et W. Snyder (2002), Cultivating Communities of Practice: a guide to managing knowledge, Harvard Business School Press 